\title{
ФИЛОСОФИЯ
}

DOI: https://doi.org/10.15688/lp.jvolsu.2021.2.18

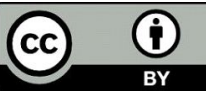

UDC 167

LBC 87.2

\section{THE ANATOMY OF RESEARCH PROCESS IN THE LIGHT OF THE PHENOMENOLOGICAL APPROACH AND THE PROBLEM OF IDENTIFICATION}

\author{
Margarita N. Kozhevnikova \\ Herzen State Pedagogical University of Russia, Saint Petersburg, Russian Federation
}

\begin{abstract}
The author undertakes a phenomenological analysis of research process on the example of the philosophical and anthropological study of education. In accordance with the phenomenological approach, the research process with its general methodological regularities is divided into three phases: 1) the preliminary phase of thematization; 2) the phenomenological phase itself, which opens up for the researcher his own direct experience of the subject, its obviousness; 3) the post-phenomenological phase. Within the boundaries of the last phase the tension between "experience" and "language" is resolved, that is, experience is expressed, an exit to the general life world apeears, an approach to certification is carried out. From this point of view, the problem of "reliability" and "identity" is raised, which is especially relevant for the phenomenological perspective. Certain phenomenological solutions to this problem were developed by Hegel and Husserl. Taking into account the existing solutions, three possible versions of the interpretations of the identity are considered: proceeding from objectivity; intersubjectivity; subjectivity. The application of phenomenological analysis to practical, scientific and philosophical fields of research allowed us to distinguish three levels of verification during certification. At the first level, experience, representation and concept come to the fore; at the second level - the requirement of unity of experience, representation and thinking; at the third - a certificate consisting of checking the stability of the trusted before the other / Other. The understanding of the latter has the meaning of a "dialectical movement", according to Hegel, and represents an "infinite horizon... of approximations", according to Husserl. These criteria are explained in the perspective of subjectivity, which reveals its possibilities when compared with interpretations based on the understanding of the true as objective (in particular, when compared with "scientific realists") and intersubjective.
\end{abstract}

Key words: research methodology, phenomenological method, dialectics, philosophical anthropology, theory of subjectivity, forms of human subjectivity.

Citation. Kozhevnikova M.N. The Anatomy of Research Process in the Light of the Phenomenological Approach and the Problem of Identification. Logos et Praxis, 2021, vol. 20, no. 2, pp. 162-171. (in Russian). DOI: https://doi.org/10.15688/lp.jvolsu.2021.2.18

УДК 167

ББК 87.2

\section{АНАТОМИЯ ИССЛЕДОВАТЕЛЬСКОГО ПРОЦЕССА В СВЕТЕ ФЕНОМЕНОЛОГИЧЕСКОГО ПОДХОДА И ПРОБЛЕМА «УДОСТОВЕРЕНИЯ»}

\section{Маргарита Николаевна Кожевникова}

Российский государственный педагогический университет им. А.И. Герцена, г. Санкт-Петербург, Российская Федерация 
Аннотация. В статье предпринят анализ исследовательского процесса (в частности, на примере философского антропологического исследования образования), трактуемого в свете феноменологического подхода. Осмысление процесса исследования с присущими ему общими методологическими закономерностями произведено в соответствии с тремя фазами, выделяемыми путем феноменологического подхода с точки зрения выявления «основы достоверного знания». Таковыми являются: 1) предварительная фаза тематизации; 2) собственно феноменологическая фаза, открывающая для исследователя собственный непосредственный опыт предмета, его очевидность; 3) постфеноменологическая фаза, разрешающая напряжение между «опытом» и «языком» и состоящая в выражении, то есть в выходе в общий жизненный мир, включающая тем самым в себя подступ к удостоверению. С этой точки зрения поднимается проблема «достоверности» и «удостоверения», особенно актуальная для феноменологической перспективы, в рамках которой задействованы определенные феноменологические решения, выработанные Гегелем и Гуссерлем. С учетом существующих решений рассмотрены возможные 3 версии трактовок удостоверения (исходящие из объективности, интерсубъективности, субъектности). В результате феноменологический анализ удостоверения в исследовании - применительно к трем областям: практической, научной и философской - позволил различить разные уровни проверки при удостоверении, в котором на первый план выходят опыт, представление и понятие; требование единства (опыта, представлений, мышления) как главный критерий и способ удостоверения, состоящий в проверке, выстоит ли удостоверяемое перед другим / Другим. Понимание последнего имеет смысл «диалектического движения» (согласно Гегелю) и представляет «бесконечный горизонт... аппроксимаций» (по Гуссерлю). Эти критерии объяснены в перспективе субъектности, обнаруживающей свои возможности при сопоставлении с версиями трактовок, исходящих из пониманий истинного как объективного (в частности, при сопоставлении с «научными реалистами») и интерсубъективного.

Ключевые слова: исследовательская методология, феноменологический метод, диалектика, философская антропология, теория субъектности, формы человеческой субъектности.

Цитирование. Кожевникова М. Н. Анатомия исследовательского процесса в свете феноменологического подхода и проблема «удостоверения» // Logos et Praxis. - 2021. - T. 20, № 2. - C. 162-171. - DOI: https://doi.org/ 10.15688/lp.jvolsu.2021.2.18

\section{Введение}

Метод всякого начинаемого исследования бывает необходимо брать в качестве предмета осмысления, а в ином случае, при техническом подходе к методу, происходит «скрывание» (по формулировке Гуссерля) [Гуссель 1992, 166-167] и во многом потеря его смысла. Достоверность составляет ключевое требование к методу исследования, а ее выявление - «удостоверение», соответственно, оказывается частью всякого метода. При этом вопрос о ней возникает, как нам кажется, относительно предъявляемых результатов исследования, то есть уже при завершении, и для его процесса может пониматься как требование соблюдения достоверности именно с точки зрения выводов. Вместе с тем «анатомия» процесса исследования способна позволить нам заметить возникновение тех чувствительных точек, в которых проблема достоверности выявляется в разных фазах процесса исследования ${ }^{1}$.

Как можно наблюдать, проблему достоверности увязывают с проблемой истины в разных версиях ее трактовок [Glanzberg web], a сейчас включают в широкое проблемное поле, охватывающее тематику истинности, верификации, правдоподобия (Verisimilitude), вероятности, эмпирически различимой истины (Empirically Discernible Truth), «примерной правды» (Approximate Truth), сходства с истиной (Truthlikeness), «близости к истине» (Closeness to the Truth), степеней подобия истинности (Degree of Truthlikeness) [Oddie web]. То, как трактуют проблему удостоверения, будет обсуждаться дальше, в завершение анализа «анатомии» исследовательского процесса.

Рассматривая исследовательский процесс и проблему удостоверения, мы обнаруживаем особые возможности феноменологического подхода для решения этих задач.

\section{Анатомия исследовательского процесса}

С точки зрения выявления «основы достоверного знания», в исследовательском процессе выделяются 3 фазы.

\section{Предварительная фаза}

Чаще при подступе к исследованию этот шаг бывает неотрефлексирован: мы как бы 
сразу имеем тематизированный предмет (в антропологическом исследовании - это «человек», в образовательно-философском - «образование») и не замечаем уже принятых определенных представлений и позиции наблюдения, что, в свою очередь, сказывается на упущении требования достоверности. В рефлексии же, предписываемой феноменологическим подходом, я вижу и учитываю момент тематизации как первый шаг или предварительную фазу, в которой обнаруживаю себя в еще-не-собственно-моем мыслимом, погруженной в понятия общего жизненного мира.

В предварительной фазе я пока еще обретаю свой предмет по имеющимся «свидетельствам» соответствующей научной области. Анализируя и сравнивая их, уточняю предмет для более строгой дальнейшей тематизации. Результатом предварительной фазы в исследовании становится ориентация изучающего в тематизации феноменов (что тематизировать и как).

\section{Собственно феноменологическая фаза}

Данная фаза состоит в собственном усмотрении исследователем предмета мышления при целенаправленном воздержании от имевшегося известного взгляда на него. Она неотделима от исследования, хотя бы она и не осознавалась. Такая неотъемлемость означает существование «естественной феноменологии»- присутствующей и в естественном мышлении, обращающемся к исследованию; а в философской и научной феноменологии эта фаза культивируется ${ }^{2}$.

То, что я «исследую» нечто - значит, что, даже имея представление о предмете, я стремлюсь изменить его, преобразуя в практическом исследовании условия своего опыта предмета, а в теоретическом исследовании производя изменения мысленно, помещая его в другие условия, в соотношения с другими предметами, сопоставляя свое представление с представлениями Других. Так «предмет для меня» (но все же не сам предмет, а мой опыт и мое представление) меняется.

Понимание состоится в поле напряжения между полюсами «опыта» и «представлений». Поэтому, когда нет действительного опыта предмета, мы чувствуем потребность отыс- кать любую основу личного переживания (видеозапись, рисунок, схему, наконец, мысленный образ). Но эта самая фаза «обращения к своему опыту» обычно не бывает замечена, как не бывает замечено, что сознание обнаруживает феномены в качестве своего содержания. Заметить и признать это - задача, которая была осмыслена и сформулирована с распознанием ее трудности в феноменологии [Гегель 1937, 25].

Значение феноменологической фазы в том, что именно она открывает основу обретаемого в исследовании достоверного знания - непосредственный опыт, очевидность ${ }^{3}$, отличную от повседневного «очевидного». То «очевидное» проникнуто предвзятостями, следуя колеей известного, смыкающегося с как бы «объективным».

Эта очевидность не существует где-то в пространстве между обсуждающими, а существует для меня (или для других Я). То есть очевидное - это ближайшее ко мне установление: то, что я наиболее близким, «непосредственным» образом устанавливаю. В этом смысле в «очевидности» на первый план выходит субъективная сторона: то, что должно стать всеобще признанным (объективным), для начала нуждается в том, чтобы быть кемmо признанным (субъективным). Очевидность содержится в индивидуальном опыте, но не как окончательная, а в качестве пребывающей в переустановлении.

Теперь феноменологический этап, если даже исходить из неизбежного присутствия понятийного мышления в любом рассмотрении, делается возможным, по словам Гегеля, при позиции «воздержания», требующей «освободиться от собственного вмешательства в имманентный ритм понятий, не вторгаться в него по произволу и с прежде приобретенной мудростью» [Гегель 1937, 36]. По Гегелю, конечно, это же - «существенный момент внимания к понятию» и, таким образом, источник становящегося постижения, при котором «наш предмет - являющееся знание» [Гегель 1937, 46].

Сформулируем еще раз феноменологический подход, как мы его принимаем для феноменологической фазы: это позиция исследования как базированного на обнаруживаемых в являющихся в сознании данных опыта, пос- 
ледовательно отличаемых от «естественных представлений» («естественной установки»), с дальнейшим вопрошанием об этих данных. На этом «пути сомнения» в результате «естественное сознание... теряет свою истину» и оказывается «нереальным знанием» [Гегель 2000, 47-48].

\section{Постфеноменологическая фаза исследования}

Эта фаза как траектория пролегает между «опытом» и «языком», связывая их. Здесь феноменологически усмотренное в «собственно-моем мышлении» должно обрести «собственно-мое выражение» в концептуализации. Особенности этой фазы всегда вели и ведут к проработке или разработке языка в философии как прямому следствию усмотрений феноменологической фазы. Но можно понять, что, при всей строгости феноменологического метода, феноменологический пуризм невозможен. Ведь выражение означает язык, а язык, будучи общим с другими и потому концептуально нагруженным, будет нести в стадии выражения и опыт, дополнительный к тому, который тематизируется. Этот опыт приходится учитывать как интерпретационную составляющую и для очищения снова переуяснять, Ad Infinitum. Признавая это, нужно осмыслять и использовать концептуальные данности языка. В частности, возможно использовать язык научной концептуальности, присутствующей в нашем фоновом знании и составляющей в систему интерпретативных релевантностей.

\section{Проблема удостоверения}

Третья фаза, состоящая в выражении, то есть в выходе в общий жизненный мир, тем самым включает в себя подступ к удостоверению, так как подразумевает его необходимость. Исследование не завершается однократным прохождением трех фаз, но, в силу вопрошания, такими же шагами движется дальше. Вопрос о методе, замеченный как основной и порождающий остальные вопросы, таков: «Чем и как удостоверяется то, что я произвожу в процессе исследования, что в итоге понимаю?»

И одной из трудностей самого феноменологического метода является то, что методо- логическое сомнение возможно, когда предполагается несомненная (пусть еще и невыявленная) область абсолютного знания ${ }^{4}$. Иначе методологическое сомнение возможно, когда остается надежда на обоснованное познание.

Рассмотрим предложенные феноменологами ответы относительно проблемы удостоверения. Гегель считает: «Нам нет необходимости прибегать к критерию» (то есть «внешнему критерию» у Гегеля), - ведь «сознание проверяет само себя». И так «мы достигаем того, что рассматриваем суть дела так, как она есть в себе самой и для себя самой» [Гегель 1937, 46-48]. В поздних работах Гуссерля отмечено, что под истинной действительностью не подразумевается очевидность и подтверждение в реальный момент текущего переживания, но сущее есть некая идеальная имманентность, и варьирование как очевидное обосновывает аподиктическое сознание всеобщности [Гуссерль 2010, 80-82].

Проблема удостоверения, исходя из содержания этого понятия, касается обнаружения усмотренного как достоверного - того, что подлинно есть как таковое, и не только для меня, но и для Других. В подходе к этой проблеме на поверхности лежит возможность следующих версий трактовок.

Первая версия: мое усмотренное соотносится с истинным, понимаемым как объективное («таково положение вещей»), с истиной как бытием (будь то взгляд материалистов, для которых это объективное бытие внешних вещей, независимых от сознания, или взгляд идеалистов, для которых это объективное бытие самого «положения вещей» как независимых идей). Происходящие из этой позиции риски впадение в диктат объективизма.

Вторая версия: усмотренное соотносится с интерсубъективным, согласованным между Другими как истинное. Таковы, например, позиции в школе прагматизма [William 1979] или широкого спектра направлений социального конструктивизма [Latour, Woolgar 1986; Knorr-Cetina 1981; Pickering 1984; Collins, Pinch 1993], разделяющих понимание достоверности как неотъемлемо зависящей от социальных факторов, достигающих крайностей позиций школы «Сильной программы» [Bloor 1984, 7594]. Трактовка значения научных терминов как «социальных институтов» (способов, при ко- 
торых состоится их успешное использование в общении внутри языкового сообщества) привела к обоснованию научных понятий как продуктов социальных переговоров, вообще не требующих фиксированного определенияния [Kusch 2002]. В рамках этой же версии (истины как интерсубъективного) может быть объяснено и существование научных парадигм Т. Куна, тяготевшего к конструктивизму [Zammito 2004].

Риски этой версии - растворение гносеологической ответственности субъекта интерсубъективности и, как следствие, - релятивизм [Seidel 2014] и сведение всеобщего к общепринятому, имеющему заметные следствия в образовании, что ведет в мире к оправданию диктата массового сознания.

Но рассмотрим обычное понимание результатов исследования (практического, научного и философского). Итак, исследование движимо неопределенностью, самой возможностью другого относительно данности. В практической области я исследую, как существует предмет (единичное), (например, обучение иностранному языку в данной группе), относительно которого есть видение и ожидание, требующие удостоверения. Оказывается, что мой опыт предмета («обучения») я, не различая, считаю им самим и не замечаю еще своего способа его понимания, представления о нем. При удостоверении исходная точка - «сам предмет», и для проверки, является ли нечто им, я действую с этим нечто так, как если бы это был «тот самый» предмет, и сверяю полученный опыт.

С чем я сверяю? Например, с бывшим у меня раньше «тождественным» опытом этого; с происходящим в других условиях моим другим опытом этого; с моим тождественным опытом, относившимся к другой вещи (таким же было обучение грамоте); с известным мне другим опытом этой вещи; с тождественным опытом совокупных Других - моего жизненного мира («так обучают все») и т. д. Обнаруживая, устаивают ли мои ожидания, я делаю выводы: достоверно ли это «тот самый» предмет, - хотя, по сути, остается вопрос, соответствует ли мой опыт тому, который, в свете моих представлений, относится к нему. Но может возникнуть вывод и о том, достоверен ли опыт, или, наконец, о моем представлении о вещи.
Исследование состоит в сопоставлении, а значит, в процессе удостоверения используется та же возможность другого, которая запустила и само исследование. А руководствуюсь в удостоверении я в основном необходимостью единства опыта - это главный критерий, - поэтому требуется тождество или аналогия с опытом, который уже был признан достоверным мною, отдельными Другими, жизненным миром. Такое удостоверение ограничивается известным опытом.

В научной области я исследую, как $c y$ ществуют определенные категории вещей: внимание от опыта (как единичного) продвигается к способу понимания вещей (категоризации), и на первый план выходит представление. Удостоверяя, я сравниваю мое представление с научными представлениями и законами как тем, что признается за истинное.

Законы утверждают обязательность (тождественность) естественного опыта, исходя из него же, и обязательность (тождественность) представлений и исходят из опыта и самих себя. Обязательный характер представлений обеспечивается рассудком и потому требует культивирования и дисциплинирования области представлений и рассудка, то есть существования науки как предназначенной для этого области и ученых как воплощающих ее в своем мышлении авторитетных Других. Так, критерий удостоверения в научном исследовании состоит в сохранении тождества и обязательности опыта и представлений всей научной области, - сверка производится с ними.

Возможность другого и Другого для удостоверения в науке уже не просто используется, но дисциплинированно культивируется. В этом смысл научных экспериментов и научного сообщества (как института диалога и критической дискуссии). Однако другое и Другой здесь еще взяты в их отдельно-внешнем существовании: как варьирование условий эксперимента, научных представлений и сопоставление.

В философском исследовании я устанавливаю существование вещей как мыслиMblx - и в результате определяю порядок мышления о вещах и мышления как такового. Отслеживая, как существует мой опыт предмета при условии моего представления о его 
категории, я прихожу к понятию, которое становится не простым «отражением» предмета или моей рефлексией над своим (категориальным) представлением о нем или саморефлексией, распознающей мой опыт не как вещь, а как мое сознание, меня самого, но снятым (по Гегелю) противоречием их как других друг другу. В работе с понятием и при его проверке я восхожу на следующий уровень обращения с тождеством и различием, преодолевая их противостояние в той логике, которую проследил Гегель в развитии понятий.

Благодаря этому в понятии нет избегания другого. Мы проводим удостоверение, проверяя, выстоит ли удостоверяемое, пропуская его через другое / Другого. Хотя процесс сверки начинается в сопоставлениях, исходящих из самости, дойдя до понятия, этот процесс уже опирается на другое. Понятие уже не разрушается другим, но питается им, как продемонстрировал Гегель. Именно так из опыта и представления оно дорастает до общего и всеобщего, вбирая в себя другое вещей, опытов, представлений. От обязательности закона, характерного для результатов научного исследования, результативное (удостоверенное) философское понятие, вобравшее противоречие в себе, переходит к нерушимой аподиктичности.

Здесь в ответе на вопрос «Как и чем производится удостоверение понятия?» я возвращаюсь к формулировкам Гегеля и Гуссерля. Гегель объяснял: «Ибо сознание есть, с одной стороны, осознание предмета, а с другой стороны, осознание самого себя». Если знание о предмете ему не соответствует, «сознание должно изменить свое знание, дабы оно согласовалось с предметом; но с изменением знания для него фактически изменяется и сам предмет <..> и проверка есть проверка не только знания, но и своего критерия» [Гегель 2000, 48]. Гегель указывал на естественный характер этой проверки, присущей сознанию как таковому. Диалектическое движение составляет суть ответа Гегеля.

И эта позиция соответствует взгляду на проблему удостоверения в русле философской антропологии с точки зрения субъектности (понимаемой как неотъемлемое свойство живого: способность быть субъектом, трактуемая преимущественно в аспекте направ- ленности $^{5}$ ), реализующейся в бесконечном разворачивании.

В подступе к диалектическому движению происходит описанный Гуссерлем процесс «пробегания, в сознании безграничности поступательного хода внутренне согласных созерцаний...» [Гуссерль 2009, 429-432, 460466]. Такой процесс объясняет развитие исследования в науке (и феноменологии) в силу «всеобщего характера научного стремления» и сомнения, ведущего к «утрате несомненности» под действием критической рефлексии.

Принимая во внимание современные позиции первой версии, утверждающие достоверность как существующую в самих вещах и ею обосновывающие научное познание, можно заметить дополнительные преимущества в точке зрения, представленной феноменологами, поскольку и сторонники «научного реализма», теряя почву под ногами, клонятся к ней. В результате опровержений прошлых научных истин новыми научными открытиями и теориями появились концепции «пессимистической индукции», или «пессимистической метаиндукции» (пессимизма, исходящего из эмпирических посылок), содержащие вывод о том, что и современные теории также являются ложными. Предполагаемое «банкротство науки» вслед за Х. Патнэмом [Putnam 1978, 22-25] можно понимать как проблему отсутствия референции для терминов относительно ненаблюдаемого, ведущую к невозможности говорить об истинности использующих их теорий. Реалисты в поиске ответов на эту проблему выработали утверждение, что более поздние теории предлагают более приближенно истинные описания своих предметов, а то, как они это делают, может быть частично прояснено при изучении способов, которыми они выстраивают свои постижения, на тех более ограниченных случаях, которые представляли их предшественники [Chakravartty 2010, 33-50; Chakravartty 2017].

Так обнаруживается тот же «бесконечный горизонт аппроксимаций», и научные реалисты оказываются уязвимыми перед вопросом: как и чем производится удостоверение научных идей. Были предложены формальные критерии: критерий относительных порядков «правдоподобия» (Verisimilitude) между теориями в данной области, которые 
устанавливаются с течением времени посредством сравнения их истинных и ложных последствий, предложенный К. Поппером и опровергнутый в позднейших работах [Miller 1974]; критерий возможных миров или «подобия», по которому условия истинности теории отождествляются с набором возможных миров, в которых она истинна, и близость к истине определяется «расстоянием» между реальным миром и мирами в этом наборе [Tichý 1976; Tichý 1978]; критерий иерархий типов, согласно которому анализируются, с одной стороны, древовидные графы типов и подтипов научных концепций и, с другой стороны, те сущности в мире, которые они предположительно репрезентируют с точки зрения сходства между узлами в тех и других [Aronson 1990]. Предложенные критерии вычленяются из общего предположения о том, что теория может считаться более приближенной к истине, чем предшествующая ей, если ранняя теория может быть описана как «ограниченный случай» более поздней. Х.Р. Пост это выразил в утверждении о том, что некоторые эвристические принципы в науке порождают теории, которые «сохраняют» успешные части своих предшественников, и в «Общем принципе соответствия», гласящем, что более поздние теории обычно учитывают успехи своих предшественников путем «разложения» их до более ранних теорий в тех областях, в которых более ранние хорошо подтверждаются [Post 1971].

Научные реалисты, изначально державшиеся первой версии трактовки проблемы удостоверения, отошли к «бесконечному горизонту аппроксимаций» со сдвигом критериев от сверки с «реальностью» к согласованию утверждений.

В качестве гипотезы предположим, что при объяснении удостоверения как происходящего в неограниченном движении феноменологическая трактовка может быть обогащена еще третьей версией, где она опирается на разворачивание субъектности [Кожевникова 2020].

Движение имманентно субъектности, и живое существует в изменении, являясь единством самого и другого. Движение сознания вызвано и производится тем, что сознание содержит и обнаруживает в себе другое, и в том подобно змее, кусающей себя за хвост, но не идет по замкнутому кругу, ведь живое существует, перерастая себя, - это раскручивающаяся спираль, в которой движение, проходя один виток до конца, заставляет двигаться виток следующего уровня.

Субъектность в своем пределе (движении в беспредельное) составляет всеобщее. Вместе с движением субъектности, удостоверение тоже строится как нескончаемое перерастание. В философском исследовании то, что бывает переподтверждено, оказывается, как было замечено выше, подлинными понятиями, которые вобрали другое и несокрушимы.

\section{Заключение}

Благодаря применению феноменологического подхода произведен анализ исследовательского процесса с выявлением в нем фаз, имеющих разное значение с точки зрения выявления «основы достоверного знания». В связи с постфеноменологической фазой исследования и в ее завершение встает проблема удостоверения, которая традиционно видится трудной для феноменологического метода. Однако в результате произведенного осмысления сути феномена «удостоверения» для случаев повседневного практического, научного и философского исследований и концептуализации трех возможных версий трактовок «удостоверения» при сопоставлении с другими возможными трактовками феноменологическая версия обнаруживает свои особые возможности, понятые в связи с теорией субъектности.

\section{ПРИМЕЧАНИЯ}

${ }^{1}$ Сама проблема достоверности обретаемого знания заняла значительное место в истории философской мысли со времен древней Греции до наших дней: у Платона, Аристотеля, скептиков, Дж. Локка, Дж. Вико, Б. Спинозы, Д. Юма, И. Канта, в разных направлениях позитивизма, феноменологии и многих других [Овчинников 2001].

${ }^{2}$ Например, помимо феноменологических методов в психологии, были развиты средства «феноменографии» [Marton 1981, 177-200].

${ }^{3}$ В определении Гуссерля: «Очевидность в наиболее широком смысле есть опыт сущего и притом сущего так, как оно есть» [Гуссерль 2010, 2427]. Здесь «очевидность» взята из сознания повсед- 
невности: это «сущее», о котором это сознание пока не может судить. Иначе, что можно было бы заметить о «сущем» для сознания повседневности, смысл его как предмета очевидности.

${ }^{4}$ Таковой для самого Гуссерля в ранний период была сфера самоданности, а в «Идеях I»- исходное дающее созерцание [Ямпольская 2013, 25]. И, по мнению Ямпольской, анализ вопроса о данности приводит к обнаружению «неявного отождествления феномена и предметности» - этой «двусмысленности феномена как корелляции явления».

${ }^{5}$ Направленность как задавание собственного направления; этот феномен лежит в основе психологических феноменов, охватываемых понятием «направленности личности» [Рубинштейн 2001, 519; Ломов 2000] и другими понятиями, относящимися к структуре направленности личности, к мотивационной сфере в целом.

\section{СПИСОК ЛИТЕРАТУРЫ}

Гегель 1937 - Гегель Г.В.Ф. Наука логики. М.: Соцэкгиз, 1937.

Гегель 2000 - Гегель Г.В.Ф. Феноменология духа. М.: Наука, 2000.

Гуссерль 1992 -Гуссерль Э. Кризис европейских наук и трансцендентальная феноменология (введение в феноменологическую философию) // Вопросы философии. 1992. № 7. С. 136-177.

Гуссерль 2009 - Гуссерль Э. Идеи к чистой феноменологии и феноменологической философии. Кн. 1. М.: Академический проект, 2009.

Гуссерль 2010 - Гуссерль Э. Картезианские медитации. М.: Академический проект, 2010.

Кожевникова 2020 - Кожевникова М.Н. Феномен субъектности: проблемы интерпретации // Ценности и смыслы. 2020. № 6 (70). С. 31-45.

Ломов 2000 - Ломов Б.Ф. Направленность личности. Субъективные отношения личности // Психология личности в трудах отечественных психологов. СПб.: Питер, 2000. С. 60-66.

Овчинников 2001 - Овчинников Н. Ф. Поиски достоверности // Философия науки. 2001. Вып. 7. C. $75-91$.

Рубинштейн 2001 - Рубинштейн М.Л. Основы общей психологии. СПб.: Питер, 2001.

Ямпольская 2013 - Ямпольская А.В. Феноменологический метод и его границы: от немецкой к французской феноменологии: автореф. дис. ... д-ра филос. наук. М.: РГГУ, 2013.

Aronson 1990 - Aronson J.L. Verisimilitude and Type Hierarchies // Philosophical Topics. 1990. № 18 (2). P. 5-28.

Bloor 1984 - Bloor D. The Strengths of the Strong Programme// Scientific rationality: The Sociological
Turn. Dordrecht; Boston; Lancaster: D. Reidel. 1984. P. 75-94.

Chakravartty 2010 - Chakravartty A. Truth and Representation in Science: Two Inspirations from Art // Beyond Mimesis and Convention: Representation in Art and Science. Dordrecht: Springer. 2010. P. 33-50.

Chakravartty 2017 - Chakravartty A. Scientific Ontology: Integrating Naturalized Metaphysics and Voluntarist Epistemology. N. Y.: Oxford University Press, 2017.

Collins, Pinch 1993 - Collins H., Pinch T. The Golem: What Everyone Should Know About Science. Cambridge: Cambridge University Press, 1993.

Glanzberg web-Glanzberg M. Truth [The Stanford Encyclopedia of Philosophy] // https://plato. stanford.edu/archives/fall2018/entries/truth.

Knorr-Cetina 1981 - Knorr-Cetina K.D. Manufacture of Knowledge. Oxford: Pergamon, 1981.

Kusch 2002 - Kusch M. Knowledge by Agreement: The Programme of Communitarian Epistemology. Oxford: Oxford University Press, 2002.

Latour, Woolgar 1986 - Latour B., Woolgar S. Laboratory Life: The Construction of Scientific Facts. Princeton: Princeton University Press, 1986.

Marton 1981 - Marton F. Phenomenography Describing Conceptions of the World Around Us // Instructional Science. 1981. № 10 (2). P. 177-200.

Miller 1974 - Miller D. Poppers Qualitative Theory of Verisimilitude// British Journal for the Philosophy of Science. 1974. № 25 (2). P. 166-177.

Oddie web-Oddie G. Truthlikeness [The Stanford Encyclopedia of Philosophy] // https:// plato.stanford.edu/archives/win2016/entries/ truthlikeness.

Pickering 1984 - Pickering A. Constructing Quarks: A Sociological History of Particle Physics. Edinburgh: Edinburgh University Press, 1984.

Post 1971 -Post H.R. Correspondence, Invariance and Heuristics: In Praise of Conservative Induction // Studies in History and Philosophy of Science. 1971. № 2. P. 213-255.

Putnam 1978 - Putnam H. Meaning and the Moral Sciences. London: Routledge, 1978.

Seidel 2014 - Seidel M. Epistemic Relativism. A Constructive Critique. Basingstoke: Palgrave Macmillan, 2014.

Tichý 1976 - Tichý P. Verisimilitude Redefined // British Journal for the Philosophy of Science. 1976. № 27 (1). P. 25-42.

Tichý 1978 - TichýP. Verisimilitude Revisited// Synthese. 1978. № 38 (2). P. 175-196.

William 1979 - William J. Pragmatism. Cambridge: Harvard University Press, 1979.

Zammito 2004 - Zammito J.H. A Nice Derangement of Epistemes: Post-Positivism in the Study of 
Science from Quine to Latour. Chicago: University ofChicago Press, 2004.

\section{REFERENCES}

Hegel G.W.F., 1937. Science of Logic. Moscow, Sotsekgiz Publ.

Hegel G.W.F, 2000. The Phenomenology of the Spirit. Moscow, Nauka Publ.

Husserl E., 1992. The Crisis of European Sciences and Transcendental Phenomenology (Introduction to Phenomenological Philosophy). Voprosy filosofii, no. 7, pp.136-177.

Husserl E., 2009. Ideas for Pure Phenomenology and Phenomenological Philosophy. Book 1. Moscow, Akademicheskiy proyekt Publ.

Husserl E., 2010. Cartesian Reflections. Moscow, Akademicheskiy proyekt Publ.

Kozhevnikova M.N., 2020. The Phenomenon of Subjectivity: Problems of Interpretation. Tsennosti i smysly, no. 6(70), pp. 31-45.

Lomov B.F., 2000. The Orientation of the Personality. Subjective Relationship of Personality. Psikhologiya lichnosti $v$ trudakh otechestvennykh psikhologov. Saint Petersburg, Piter Publ., pp. 60-66.

Ovchinnikov N.F., 2001. The Search for Authenticity. Filosofiya nauki, vol. 7, pp. 75-91.

Rubinshteyn M.L., 2001. Fundamentals of General Psychology. Saint Petersburg, Piter Publ.

Yampolskaya A.B., 2013. The Phenomenological Method and its Boundaries: From German to French Phenomenology. Dr. philos. sci. abs. dis. Moscow, RGGU.

Aronson J.L., 1990. Verisimilitude and Type Hierarchies. Philosophical Topics, no. 18 (2), pp. 5-28.

Bloor D., 1984. The Strengths of the Strong Programme. Scientific rationality: The sociological turn. Dordrecht; Boston; Lancaster, D. Reidel Publ., pp. 75-94.

Chakravartty A., 2017. Scientific Ontology: Integrating Naturalized Metaphysics and Voluntarist Epistemology. New York, Oxford University Press.

Chakravartty A., 2010. Truth and Representation in Science: Two Inspirations from Art. Beyond
Mimesis and Convention: Representation in Art and Science. Dordrecht, Springer Publ., pp. 33-50.

Collins H., Pinch T., 1993. The Golem: What Everyone Should Know About Science. Cambridge, Cambridge University Press.

Glanzberg M. Truth. The Stanford Encyclopedia of Philosophy. URL: https://plato.stanford.edu/ archives/fall2018/entries/truth.

Knorr-Cetina K.D., 1981. Manufacture of Knowledge. Oxford, Pergamon Publ.

Kusch M., 2002. Knowledge by Agreement: The Programme of Communitarian Epistemology. Oxford: Oxford University Press.

Latour B., Woolgar S., 1986. Laboratory Life: The Construction of Scientific Facts. Princeton, Princeton University Press.

Marton F., 1981. Phenomenography - Describing Conceptions of the World Around Us. Instructional Science, no. 10 (2), pp. 177-200.

Miller D., 1974. Poppers Qualitative Theory of Verisimilitude. British Journal for the Philosophy of Science, no. 25 (2), pp. 166-177.

Oddie G. Truthlikeness. The Stanford Encyclopedia of Philosophy. URL: https://plato.stanford.edu/ archives/win2016/entries/truthlikeness.

Pickering A., 1984. Constructing Quarks: A Sociological History of Particle Physics. Edinburgh, Edinburgh University Press.

Post H.R., 1971. Correspondence, Invariance and Heuristics: In Praise of Conservative Induction. Studies in History and Philosophy of Science, vol. 2, pp. 213-255.

Putnam H., 1978. Meaning and the Moral Sciences. London: Routledge Publ.

Seidel M., 2014. Epistemic Relativism. A Constructive Critique. Basingstoke, Palgrave Macmillan Publ.

Tichý P., 1976. Verisimilitude Redefined. British Journal for the Philosophy of Science, no. 27 (1), pp. 25-42.

Tichý P., 1978. Verisimilitude Revisited. Synthese, no. 38 (2), pp. 175-196.

William J., 1979. Pragmatism. Cambridge, Harvard University Press.

Zammito J.H., 2004. A Nice Derangement of Epistemes: Post-Positivism in the Study of Science from Quine to Latour. Chicago: University of Chicago Press. 
М.Н. Кожевникова. Анатомия исследовательского процесса в свете феноменологического подхода

\section{Information About the Author}

Margarita N. Kozhevnikova, Candidate of Sciences (Philosophy), Head of the Research Laboratory of Problems of Personality Social Support, Herzen State Pedagogical University of Russia, Reki Moyki Emb., 48, 191186 Saint Petersburg, Russian Federation, mkozhevnikova1@gmail.com, https://orcid.org/0000-0002-9093-4936

\section{Информация об авторе}

Маргарита Николаевна Кожевникова, кандидат философских наук, заведующая научноисследовательской Лабораторией проблем социальной поддержки личности, Российский государственный педагогический университет им. А.И. Герцена, наб. реки Мойки, 48, 191186 г. СанктПетербург, Российская Федерация, mkozhevnikova1@gmail.com, https://orcid.org/0000-0002-9093-4936 